\section{Marine Biology}

Volume 151, Number 5 / juin 2007

http://dx.doi.org/10.1007/s00227-007-0624-1

(c) 2007 Springer
Archimer, archive institutionnelle de l'Ifremer http://www.ifremer.fr/docelec/

The original publication is available at http://www.springerlink.com

\title{
Anchovy (Engraulis encrasicolus ) egg density measurements in the Bay of Biscay: evidence for the spatial variation in egg density with sea surface salinity
}

\author{
Anne Goarant ${ }^{1}$, Pierre Petitgas ${ }^{1,{ }^{*}}$ and Paul Bourriau ${ }^{1}$
}

(1) Department Ecology and Models for Fisheries, IFREMER, rue de l'île d'Yeu, BP22105, 44311 cdx 3, Nantes, France

*: Corresponding author : pierre.petitgas@ifremer.fr

\begin{abstract}
:
Knowledge of the pelagic vertical distribution of fish eggs is central for several aspects of fisheries science including fisheries recruitment and egg production studies. In modelling egg vertical distributions, variation in fish egg density is an important issue. Though variation in egg density between individual eggs has been reported, evidence for significant spatial variation in egg density is novel. The present study provides evidence that egg density of anchovy (Engraulis encrasicolus) varies spatially across spawning sites in the Bay of Biscay, depending on the regional scale variation in sea water properties due to river discharge. We measured the density of the eggs using a density gradient column at 17 stations in 2005 and 2006 as well as their diameter. At station, the variability in the individual egg density was statistically distributed according to a Gaussian probability function. Significant variation in the mean egg density was observed across stations. Mean egg density displayed a significant correlation with sea surface salinity. Results are discussed in light of the mechanisms determining the egg density.
\end{abstract}

Keywords: Density gradient column, fish egg density, anchovy, Bay of Biscay 


\section{Introduction}

\subsection{Importance of fish egg density in predicting vertical egg distribution.}

Knowledge of the pelagic vertical distribution of fish eggs is central for several aspects of fisheries science, such as (i) estimating initial conditions for larvae drift and survival models, (ii) estimating ambient developmental temperature for monitoring stocks with egg production methods and (iii) defining appropriate sampling methodologies. The vertical distribution of pelagic eggs is determined by a set of interacting biological and physical processes (Sundby, 1991), namely the properties of the eggs (density, diameter) and that of the ambient sea water (density, viscosity, turbulence). The vertical distribution of pelagic eggs is difficult to access to in the field and models have been developed (Sundby, 1983; Westgård, 1989; Petitgas et al., 2006). Model limitations reside in the biological knowledge more than in the model parameterisation. In particular, variability in egg density at different spatial scales is not well known.

\subsection{How and when is egg density is determined?}

Most marine Teleost fishes have pelagic eggs that show excess buoyancy in comparison to surface sea water (e.g., Mellinger, 1994, who envisage this property as an adaptation to marine life). The egg density is determined in the ovary prior to the ovulation (Nissling et al., 2003) during the process of oocyte hydration. Oocyte hydration involves yolk proteolysis resulting in increasing oocyte osmolarity (Craik and Harvey, 1987) and water passage through the vitelling membrane using molecular water channels (Fabra et al., 2005). Oocyte hydration is then a mechanism by which the egg density can be regulated resulting in adjusting the egg buoyancy to the ambient sea water conditions experienced by the spawning adults. Observed variability in egg density would then result from such adjustment.

\subsection{Reported variations in egg density.}

Egg density was reported to vary with sea water density between the Baltic and the North Sea (e.g., Nissling and Vallin, 1996; Solemdal, 1971) as well as seasonally in the English Channel (Coombs et al., 1985). Egg density was further reported to vary between years with sea water density based on indirect estimates of egg density using vertical modelling (Petitgas et al., 2006). In the case of sardine, sprat and anchovy, individual egg density was reported to be constant throughout the egg life span from fertilisation to just before hatching (Coombs et al., 1985; Coombs et al., 2004). Variation at station in the individual egg density was assumed Gaussian random in vertical models (Sundby, 1983). Spatial variation in egg density across spawning grounds has never been reported, e.g., between station variation has never been reported to be greater than the within-station variability.

\subsection{The present study.}

The Bay of Biscay offers a mosaic of different hydrological structures (Koutsikopoulos and Le cann, 1996; Planque et al., 2006) ranging from oceanic conditions to coastal waters under the influence of river discharge, all in which anchovy spawns (Motos et al., 1996). In the present study, we measured anchovy egg density onboard in different sea surface water conditions in the Bay of Biscay. With these measurements we investigated whether the at station variation between individual eggs was Gaussian. We also investigated whether the egg density varied in space across different spawning grounds showing different hyrological characteristics. Sea surface salinity and density were naturally chosen as covariates based on the spawning biology of the anchovy. Effectively, anchovy was known to spawn during night time at surface (purse seine fishery; Motos, 1996; Palomera, 1991) and sea water density or salinity potentially affected the hydration of the oocytes (see above). 


\section{Material and methods}

Anchovy eggs were collected in May-June 2005 and 2006 onboard the R/V Thalassa (Table 1, Fig. 1) during IFREMER's yearly fisheries pelagic acoustic monitoring survey (Pelgas series).

\subsection{Anchovy egg sampling}

At each station prior to icthyoplankton sampling, a CTD (conductivity, temperature, depth) profile was performed from the surface to the bottom or a maximum depth of 200 meters. The depth of the bottom of the thermocline was then determined, which defined the maximum depth of the icthyoplankton sampling. Eggs were collected using the Carré net developed by IFREMER (Bourriau, 1991), which is a squared aperture $\left(1^{\star 1} \mathrm{~m}^{2}\right)$ net. The net was hauled at 2 knots. In 2005, the net was hauled horizontally at sub-surface (3 m depth). In 2006, the net was hauled from surface to the bottom of the thermocline and back to the surface, resulting in a $V$ underwater trajectory. In shallow water (e.g., $20 \mathrm{~m}$ ) to ensure sufficient sea water filtration, the $V$ shape haul was repeated twice resulting in a $\mathrm{W}$ underwater haul trajectory. Average length tow was 9 minutes and average filtered volume was 505 cubic meters (Table 1). Because measurements were performed on living eggs, precautions were taken to avoid damaging the eggs when taking the sample from the collector of the net, in particular the production of bubbles and turbulence. The sample was brought to the laboratory on board where eggs were manipulated individually with sea water washed instrumentation. At each station, an average of 70 eggs were sorted from the sample. 50 served for the measurement of their density and 20 for that of their size. Egg measurements were made irrespective of egg developmental stage as anchovy egg parameters (density and size) were reported to be constant all along the egg life span (Coombs et al., 2004).

\subsection{Egg diameter measurements}

The anchovy egg is a prolate ellipsoid with one big axis of diameter $2 \mathrm{a}$ and two small axes of equal diameter 2b (Boyra et al., 2003; Coombs et al., 2004). Diameters 2a and $2 \mathrm{~b}$ were measured on alive eggs to a precision of $0.1 \mathrm{~mm}$ using a binocular equipped with a calibrated micro ruler at $\times 120$ magnification. The egg volume considered was that of the ellipse of revolution around the big axis. The radius of the equivalent sphere (egg size) was then derived: $\left(a b^{2}\right)^{1 / 3}$.

\subsection{Calibration of the density gradient column}

Measurements of egg density were performed using the density gradient column apparatus of Coombs (1981). The column is filled with a continuously graded solution of sea water salts such that an egg introduced in the column settles to a position where it is in hydrostatic equilibrium. The column being graduated and the density gradient being calibrated, the reading of the settlement position of the egg corresponds to the measurement of its density. Five floats of known densities ranging from 21.3 to 27.0 sigma-t served to calibrate the density gradient. The column was graduated every $2 \mathrm{~mm}$ from 0 to $700 \mathrm{~mm}$. The floats settlement positions were read with a precision of $1 \mathrm{~mm}$. The float positions $(\mathrm{mm})$ were linearly regressed on the float densities (sigma-t) and the regression line served as calibration line (the central part of the density gradient where eggs settled was always linear). At least one hour prior to the introduction of the eggs in the column, a new density gradient was made and calibrated. Station specific regression lines all had $\mathrm{R}$-squared close to 0.99 and thus the error in calibrating the gradient was neglected. The average slope of the calibration lines was such that a $10 \mathrm{~mm}$ height interval in the column corresponded to 0.1 sigma-t. The density gradient column can resolve differences in density of 0.04 sigma-t at maximum accuracy (Coombs, 1981). The density column gradient was kept at a thermostatic controlled temperature of $15^{\circ} \mathrm{C}$.

\subsection{Density gradient column readings}

At each station, 50 eggs were introduced alive one by one in the density gradient column, allowing for the direct estimation of the frequency distribution of the egg density. Reading was difficult when more than 50 eggs were introduced in the column. The number of settled eggs were counted every $10 \mathrm{~mm}$ interval. The precision measure of individual egg density was thus 0.1 sigma-t. Four 
readings were made at one hour interval, the first one starting 30 minutes after the introduction of eggs in the column. A table $n[x, t]$ of egg counts at height $\mathrm{x}$ and time $\mathrm{t}$ was obtained. The egg density was derived using the calibration equation: $\operatorname{dens}(x)=\mathrm{a} x_{\text {egg }}+\mathrm{b}$. The frequency distribution of the height of the eggs in the column was estimated by:

$$
f(x)=\sum_{t} n[x, t] / \sum_{x} \sum_{t} n[x, t]
$$

The mean density was: $m=\sum_{x} f(x) \operatorname{dens}(x)$, and the variance:

$$
v=\sum_{x} f(x) \operatorname{dens}(x)^{2}-m^{2}
$$

During the reading experiments, the calibration floats didn't change their position meaning that the density gradient stayed unchanged.

\subsection{Data analysis}

At each of the stations we tested whether the frequency distribution in the individual egg density was Gaussian using the Shapiro-Wilk test (Shapiro and Wilk, 1965). Significant variation in mean egg density across stations was tested against intra-station variation in individual egg density using ANOVA and F-test. Across station variation in mean egg density was then regressed on surface $(3 \mathrm{~m})$ sea water density and salinity, which were measured at each station using the CTD profiler. A parametric bootstrap procedure (Manly, 1997) was set up to test for significance in the slope of the regression as well as derive its confidence limits.

\section{Results}

\subsection{Estimate of mean density and its precision}

The anchovy egg density measurements were compiled for each station in Table 2. Mean egg density at station varied between 22.51 and 25.27 sigma-t with a mean of 24.14 sigma-t. To compute the precision on the at station mean density, we considered two sources of variation. Let $\sigma_{e}$ denote the standard deviation in individual egg density at any given station and $\sigma_{r}$ the standard deviation in the measurement error. Considering that $n$ eggs in the column provided $n$ non-correlated measures of density, the standard deviation of the mean was: $\sqrt{\left(\sigma_{r}{ }^{2}+\sigma_{e}{ }^{2}\right) / n}$. The standard deviation in individual egg density was on average $\sigma_{e}=0.62$ sigma-t. Individual egg density was measured per $l=0.1$ sigma-t intervals. Considering that eggs were uniformly distributed in the intervals, the standard deviation for the reading error was $\sigma_{r}=l / \sqrt{12}=0.029$. The precision on the estimate of the mean density at any given station was thus 0.088 sigma-t.

\subsection{Within and between station variation in egg density}

The variation between individual egg densities within any sample was larger than measurement precision. At each station the frequency distribution of the egg density was visually close to a Gaussian probability distribution (Figs. $2 \mathrm{a}$ and $2 \mathrm{~b}$ ). The normality of the distribution was tested using the Shapiro-Wilk test. At the risk $\alpha=0.05$, the Gaussian distribution was rejected for only two stations, namely J0321 and J0333. Accepting a higher risk $\alpha=0.01$, the Gaussian distribution was never rejected for none of the stations. Thus it was concluded that the variation in individual egg density at any given station had a Gaussian probability distribution. Mean egg density varied across stations between 23.08 and 25.27 sigma-t. The F-test of the ANOVA (Df = 16,830, p-value $=2.2 \mathrm{e}-16$ ) concluded that the variation in the mean egg density was significant between stations. 


\subsection{Relationship between surface salinity and egg density}

To further explain the across station variation in mean egg density, mean egg density was linearly regressed on surface ( $3 \mathrm{~m}$ depth) sea water density and salinity. Sea water density and salinity were both significant covariates but the regression on salinity was better fitted as shown by smaller residuals, a higher F-statistic and a higher $\mathrm{R}^{2}$ (Table 3). The regression on salinity explained $73 \%$ of total variance.

\subsection{Bootstrapping the regression of mean egg density vs. surface salinity.}

A parametric bootstrap was used to estimate the probability distribution of the regression slope. The bootstrap procedure mimicked the estimation of the at station mean egg density and its regression on salinity. For each station 50 egg density values were drawn at random from a Gaussian distribution with mean and variance equal to that experimentally estimated at the considered station. For each station, the 50 values were averaged. The averages were then linearly regressed on the surface salinity. This scheme was repeated 1000 times, providing 1000 slope estimates and allowing for the estimation of the probability distribution of the regression slope (Fig. 3). A Student t-test showed that the slope differed significantly from 0 ( $p$-value $=2.2 e-16)$. The distribution of the slope was symmetrical (Fig. 4). The mean slope was 1.12 sigma-t per salinity unit and the standard deviation was 0.04 . The slope had thus a precision CV of 0.0357 . The $95 \%$ confidence interval of the slope was [1.04, 1.20].

\section{Discussion}

\subsection{Estimated values of egg density and size}

The present study provided a data set on anchovy egg density under different hydrological conditions. Egg density values measured by previous authors in the Bay of Biscay (Boyra et al., 2003: $23.264 \pm 0.629$ sigma-t ; Coombs et al., $2004: 23.1$ sigma-t) were in the range of our results (22.51 to 25.27 sigma-t). Mean egg diameters were $1.4 \mathrm{~mm}$ and $0.6 \mathrm{~mm}$ resulting in an equivalent diameter of $0.4 \mathrm{~mm}$. These values agreed with that of previous authors for anchovy in the Bay of Biscay (Boyra et al., 2003: $1.4 \mathrm{~mm} \times 0.6 \mathrm{~mm}$; Coombs et al., 2004: $1.49 \mathrm{~mm} \times 0.69 \mathrm{~mm}$ ). Because of the link between egg density and sea water salinity, the full comparison between density values would require knowledge on the hydrological conditions. Therefore any measure of egg density in the field should include the acquisition of ambient sea water hydrological characteristics.

\subsection{Estimation of the egg density distribution}

Our measurements allowed for the direct estimation of the probability distribution of the egg density for vertical samples. The hypothesis of a Gaussian distribution made by Sundby (1991) was validated (Fig. 2). The protocol used provided satisfactory precision on the mean egg density estimate (standard deviation of 0.088 sigma-t). For comparison, we can compute the precision on the mean for another protocol in which a small number of alive eggs (e.g., 10) would be introduced in the density gradient column and individual egg density measured with high precision (every 1 $\mathrm{mm}$ corresponding to 0.02 sigma-t). That protocole would provide a standard deviation on the mean estimate of 0.196 sigma-t $\left(\sigma_{e}=0.62, \sigma_{r}=0.006, \mathrm{n}=10\right.$ ). Because variability in individual egg density was much larger than measurement precision, our protocol (greater number of eggs and lower precision in individual measure) was effective in estimating the sample frequency distribution in egg density and its mean. 


\subsection{Sampling effect}

In 2005, eggs were collected by subsurface hauls positioned at $3 \mathrm{~m}$ depth while in 2006 eggs were sampled by vertically integrated hauls from surface to the bottom of the thermocline. We would expect that the change in sampling methodology would have an effect on the obtained egg density distribution and the relationship between salinity and mean egg density. We compared the previous model (egg density surface salinity) to a model that in addition to salinity took into account, as factor, a sampling effect (egg density surface salinity + sampling). The slopes of the two models were not significantly different (ANOVA, $F_{15,14}=0.9503$, $p$-value $=0.3462$ ). Thus the two different sampling methods did not affect our results. The $3 \mathrm{~m}$ depth samples contained similar eggs than the vertically integrated hauls, probably because of sufficient turbulent mixing of the eggs in the water column.

\subsection{Egg size measurement precision}

We did not find any significant variation in egg equivalent radius across stations nor with egg density or sea water salinity (not shown), though one would expect egg density, egg size and sea water salinity to be related. But depending on the measurement precision on egg density and size as well as the range in the biological response in these parameters, the relationships may not always be observable experimentally. Relationships have been reported in areas where the range of variation in the parameters was large. In the Black sea on anchovy, Gordina et al. (1997) reported egg size to vary with sea water salinity depending on spawning areas. In the Baltic sea on sprat, Nissling et al. (2003) evidenced a relationship between egg size and egg density. In their study, the range of variation in the density was 5 sigma-t and that in the size was $0.2 \mathrm{~mm}$. In the present study, the range of variation in the density was 3 sigmat-t and that in the size was smaller than $0.1 \mathrm{~mm}$ (our measurement precision). Therefore for anchovy in the Bay of Biscay a measurement precision of $0.1 \mathrm{~mm}$ on egg diameters is thought insufficient to evidence any significant variation in egg size.

\subsection{Relationship between egg density and sea water salinity}

The egg density varied across spawning grounds with ambient sea water salinity. This agreed with the biological process of oocyte hydration as described in the literature (see introduction). The relationship was linear between egg density and sea water salinity. But outside the range of observed salinity one could expect a sigmoid-like relationship where the egg density would reach a low and a high sill at low and high salinity. The linear regression left $27 \%$ unexplained variance in mean egg density. This part of the variability could be due to other factors than oocyte hydration, in particular the chemical composition of the vitellus (maternal and / or environmental effects) or the advection / diffusion of the eggs. The fact that the egg density was well correlated to the ambient sea water salinity at the location of the egg sampling would tend to argue that the drifts of the eggs from their spawning location to their sampling location was within the spatial variation of the salinity.

\subsection{Consequences of our results}

The positive correlation between the egg density and the sea water salinity can be seen as the result of an adaptive spawning process, which will tend to maintain the eggs in the surface layers of the ocean whatever its hydrological variability. The average egg was in general positively buoyant with an excess buoyancy of 0.7 sigma-t in comparison to sea water surface density (Tables 1 and 2). The excess of buoyancy was similar in value to the standard deviation in the individual egg variability $\left(\sigma_{e}=0.62\right)$. The frequency distribution in the individual egg density being Gaussian one would expect a fraction of eggs to be negatively buoyant at sea surface (in our case 16\%). The range in the individual variability of the egg density is therefore very important to know as it results in smoothing the egg vertical distribution across a higher depth range. Sensitivity analyses have shown that the egg density is a crucial model parameter controlling the egg vertical distribution (e.g., Petitgas et al., 2006) and particle 2D Lagrangian drift (e.g., Parada et al., 2003). The 
monitoring of the frequency distribution in the individual egg density is thought necessary if any reliable modelling of the vertical egg distribution is to be achieved. The present work suggested a protocol for doing so.

\section{Acknowledgements}

The authors would like to thank Jacques Massé (Ifremer in Nantes) for organising the PelGas cruise in a way that allowed for the egg sampling and density measurements. Daniel Halgand (Ifremer in Nantes) contributed to the measurements on board. The study was supported by the Ifremer project FOREVAR affiliated to GLOBEC, and the European projects FISBOAT and UNCOVER. 


\section{References}

Bourriau P (1991) The "Carré Net". ICES CM 1991/L:53

Boyra G, Rueda L, Coombs S, Sundby S, Adlandsvisk B, Santos M and Uriarte A (2003) Modelling the vertical distribution of eggs of anchovy (Engraulis encrasicolus) and sardine (Sardina pilchardus). Fisheries Oceanography, 12(4/5): 381-395

Coombs S (1981) A density-gradient column for determining the specific gravity of fish eggs with particular reference to eggs of mackerel Scomber scombrus. Marine Biology, 63: 101-106

Coombs S, Fosh C and Keen M (1985) The buoyancy and vertical distribution of eggs of sprat (Sprattus sprattus) and pilchard (Sardina pilchardus). J. Mar. Biol. Assoc. U.K., 65: 461-474

Coombs S, Boyra G, Rueda L, Uriarte A, Santos M, Conway D and Halliday N (2004) Buoyancy measurements and vertical distribution of eggs of sardine (Sardina pilchardus) and anchovy (Engraulis encrasicolus). Marine Biology, 145: 959-970

Craik J and Harvey S (1987) The causes of buoyancy in eggs of marine teleosts. Journal of the Marine Biological Association of the UK, 67: 169-182

Fabra M, Raldúa D, Power DM, Deen PMT, Cerdà J (2005) Marine fish egg hydration is aquaporinmediated. Science, 307: 545-545

Gordina AD, Nikolskiy VN, Niermann U, Bingel F, Subbotin AA (1997) New data on the morphological differences of anchovy eggs (Engraulis encrasicolus) in the Black Sea. Fisheries Research, 31: 139-145

Koutsikopoulos C, Le Cann B (1996) Physical processes and hydrological structures related to the Bay of Biscay ancvhovy. Scientia Marina, 60(Suppl.2): 9-19

Mellinger J (1994) La flottabilité des oeufs de téléostéens. Année biologique. 33(3) : 117-138

Manly B (1997) Randomization, bootstrap and monte carlo methods in biology. Chapman and Hall,London, $2^{\text {nd }}$ Ed., $399 p$

Motos L (1996) Reproductive biology and fecundity of the Bay of Biscay anchovy population (Engraulis encrasicolus L.). Scientia Marina, 60(Suppl.2): 195-207

Motos L, Uriarte A, Valencia V (1996) The spawning environment of the Bay of Biscay anchovy (Engraulis encrasicolus L.). Scientia Marina, 60(Suppl.2): 117-140

Nissling A, Vallin L (1996) The ability of cod eggs to maintain neutral buoyancy and the opportunity for survival in fluctuating conditions in the Baltic Sea. J. Fish Biol., 48: 217-227

Nissling A, Müller A, Hinrichsen HH (2003) Specific gravity and vertical distribution of sprat eggs in the Baltic Sea. Journal of fish biology, 63: 280-299

Palomera I (1991) Vertical distribution of eggs and larvae of Engraulis encrasicolus in stratified waters of the western Mediterranean. Marine Biology, 111: 37-44

Parada C, van der Lingen C, Mullon C, Penven P (2003) Modelling the effect of buoyancy on the transport of anchovy (Engraulis capensis) eggs from spawning to nursery grounds in the southern Benguela : an IBM approach. Fisheries Oceanography, 12: 170-184

Petitgas P, Magri S, Lazure P (2006) One-dimensional biophysical modelling of fish egg vertical distribution in shelf seas. Fisheries Oceanography, 15: 413-428

Planque B, Lazure P, Jegou AM (2006) Typology of hydrological structures modelled and observed over the Bay of Biscay shelf. Scientia Marina, 70(Suppl.1): 43-50

Shapiro SS, Wilk MB (1965) An analysis of variance test for normality (complete samples), Biometrika, 52, 3 and 4, pages 591-611

Solemdal P (1971) Prespawning flounders transferred to different salinities and the effects on their eggs. Vie et milieu, 1 (Suppl. 22) : 409-423

Sundby S (1983) A one-dimensional model for the vertical distribution of pelagic fish eggs in the mixed layer. Deep Sea Res., 30 (6A): 645-661

Sundby S (1991) Factors affecting the vertical distribution of eggs. ICES Mar. Sci. Symp., 192 : 3338

Westgård T (1989) Two models for the vertical distribution of pelagic fish eggs in the turbulent upper layer of the ocean. Rapp. P.-V. Réun. Const. Int. Explor. Mer, 191: 195-200 
Table 1: Characteristics of the sampling sites in 2005 and 2006. Tow duration is in minute and filtered volume in cubic meter. Sea surface temperature $\left({ }^{\circ} \mathrm{C}\right)$, salinity and density (sigma-t) are the CTD probe values at $3 \mathrm{~m}$ depth.

\begin{tabular}{c|cccccccc} 
& \multicolumn{1}{c}{ Sow } & Filtered & Temperatur & Tow \\
Station & Date & Latitude & Longitude & $\begin{array}{c}\text { duration } \\
\text { volume }\end{array}$ & $\begin{array}{c}\text { e } \\
\text { Salinity }\end{array}$ & Density \\
\hline J0315 & $26 / 05 / 2005$ & 44.04 & -1.59 & 5 & 559 & 17.62 & 34.78 & 25.20 \\
J0317 & $26 / 05 / 2005$ & 44.33 & -1.40 & 15 & 948 & 16.92 & 34.80 & 25.38 \\
J0321 & $29 / 05 / 2005$ & 45.18 & -1.31 & 10 & 243 & 15.79 & 33.21 & 24.41 \\
J0322 & $29 / 05 / 2005$ & 45.35 & -1.36 & 10 & 489 & 17.63 & 32.91 & 23.76 \\
J0324 & $29 / 05 / 2005$ & 45.55 & -1.38 & 10 & 399 & 14.84 & 32.79 & 24.30 \\
J0325 & $29 / 05 / 2005$ & 45.57 & -1.58 & 8 & 521 & 16.85 & 33.84 & 24.66 \\
J0326 & $29 / 05 / 2005$ & 45.69 & -1.50 & 10 & 716 & 16.69 & 33.30 & 24.28 \\
J0330 & $30 / 05 / 2005$ & 45.55 & -2.11 & 15 & 1025 & 16.91 & 34.70 & 25.30 \\
J0333 & $30 / 05 / 2005$ & 45.81 & -1.64 & 8 & 605 & 16.17 & 34.17 & 25.07 \\
K0310 & $09 / 05 / 2006$ & 45.45 & -1.92 & 10 & 882 & 14.89 & 33.68 & 24.98 \\
K0367 & $21 / 05 / 2006$ & 45.25 & -1.43 & 8 & 338 & 16.02 & 33.69 & 24.74 \\
K0369 & $23 / 05 / 2006$ & 45.23 & -2.00 & 8 & 509 & 15.46 & 34.51 & 25.49 \\
K0374 & $24 / 05 / 2006$ & 45.05 & -2.45 & 8 & 513 & 15.37 & 34.61 & 25.59 \\
K0377 & $25 / 05 / 2006$ & 44.00 & -1.38 & 8 & 260 & 17.52 & 33.60 & 24.31 \\
K0379 & $25 / 05 / 2006$ & 44.03 & -1.43 & 6 & 174 & 17.60 & 33.87 & 24.51 \\
K0382 & $26 / 05 / 2006$ & 44.07 & -1.46 & 8 & 241 & 17.71 & 34.31 & 24.81 \\
K0393 & $27 / 05 / 2006$ & 44.03 & -1.39 & 6 & 160 & 17.36 & 34.11 & 24.74
\end{tabular}


Table 2 : Anchovy egg density measurements at station. Mean egg density ( $m$ in sigma-t), standard deviation ( $\mathrm{e}$ in sigma-t) and CV ( $\mathrm{e} / \mathrm{m}$ ) of individual egg density. The bottom line gives the mean value for each of the columns.

\begin{tabular}{c|cccc} 
Station & mean & $\sigma_{\mathrm{e}}$ & $\mathrm{CV}$ & No Eggs \\
\hline J0315 & 25.27 & 0.46 & 0.02 & 31 \\
J0317 & 24.92 & 0.40 & 0.02 & 52 \\
J0321 & 23.27 & 0.72 & 0.03 & 85 \\
J0322 & 23.59 & 0.53 & 0.02 & 39 \\
J0324 & 22.51 & 0.74 & 0.03 & 46 \\
J0325 & 23.08 & 0.54 & 0.02 & 49 \\
J0326 & 23.72 & 0.69 & 0.03 & 59 \\
J0330 & 25.14 & 0.85 & 0.03 & 45 \\
J0333 & 23.89 & 0.32 & 0.01 & 46 \\
K0310 & 24.67 & 0.86 & 0.03 & 50 \\
K0367 & 23.22 & 0.68 & 0.03 & 80 \\
K0369 & 24.89 & 0.78 & 0.03 & 41 \\
K0374 & 25.14 & 0.56 & 0.02 & 36 \\
K0377 & 23.74 & 0.69 & 0.03 & 46 \\
K0379 & 24.15 & 0.48 & 0.02 & 50 \\
K0382 & 24.72 & 0.60 & 0.02 & 49 \\
K0393 & 24.53 & 0.58 & 0.02 & 58 \\
\hline average & 24.14 & 0.62 & 0.03 & 51
\end{tabular}

Table 3: Summary table comparing the linear regression models of egg density on sea water surface $(3 \mathrm{~m})$ salinity and density.

\begin{tabular}{|c|c|c|c|c|c|c|}
\hline Covariate & Parameter & Estimate & $\begin{array}{c}\text { Standar } \\
\text { d error }\end{array}$ & $p$ value & $\begin{array}{c}\mathrm{F} \\
\text { statistic }\end{array}$ & $\mathrm{R}^{2}$ \\
\hline \multirow[t]{3}{*}{ Salinity } & $\beta_{0}$ (intercept) & -13.7919 & 5.959 & 0.03520 & & \\
\hline & $\beta_{1}$ (slope) & 1.1179 & 0.176 & 0.00001 & & \\
\hline & & & & & $\begin{array}{c}40.54 \\
(\mathrm{df}=1.15 \\
)\end{array}$ & 0.7299 \\
\hline \multirow[t]{3}{*}{ Density } & $\beta_{0}$ (intercept) & -6.8072 & 7.0957 & 0.3525 & & \\
\hline & $\beta_{1}$ (slope) & 1.2482 & 0.2861 & 0.00056 & & \\
\hline & & & & & $\begin{array}{c}19.03 \\
(\mathrm{df}=1.15 \\
)\end{array}$ & 0.5593 \\
\hline
\end{tabular}



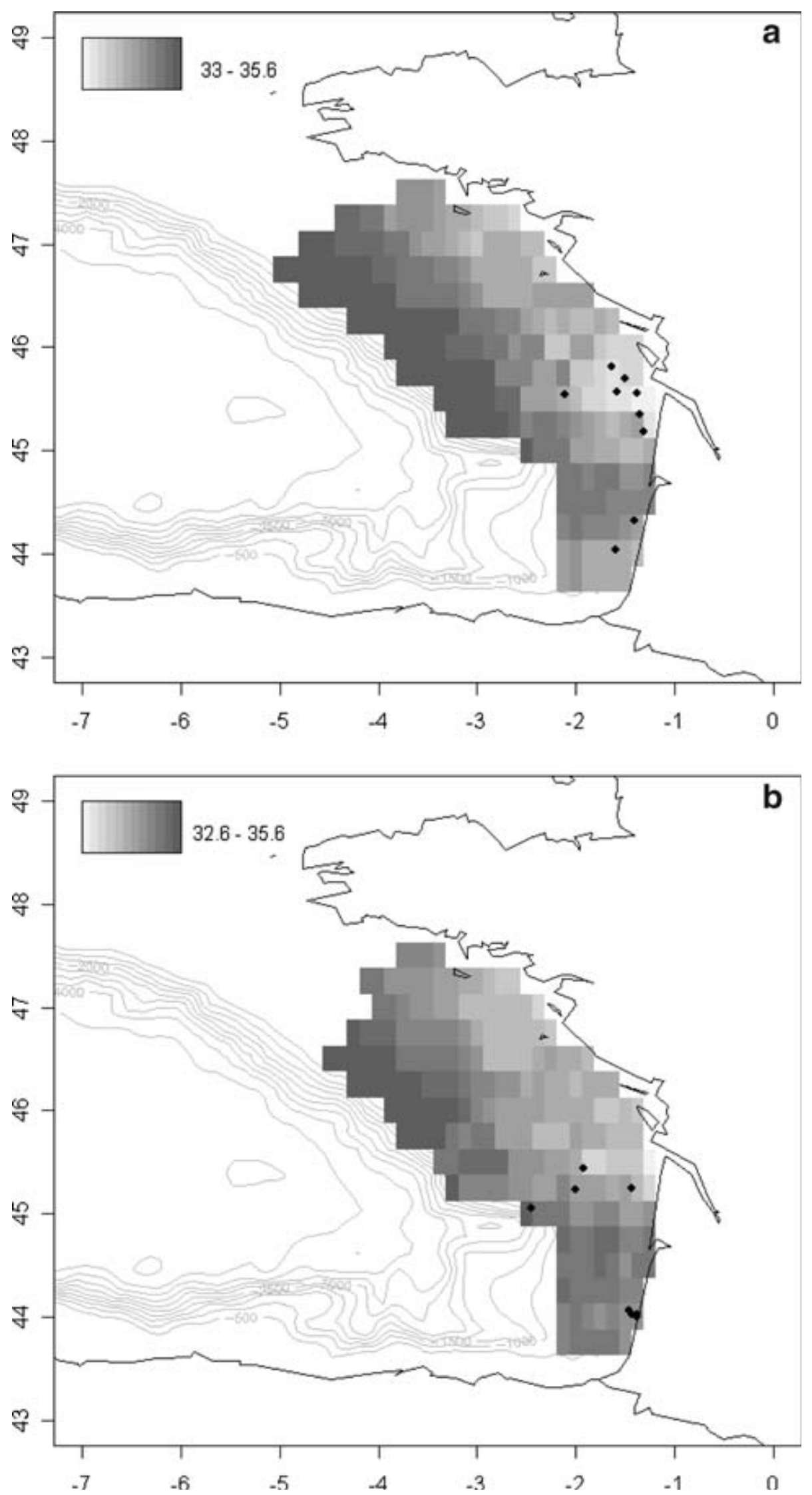

Figure 1: Map of the sampling stations. Circles are for the 2005 stations and triangles for the 2006 stations. 

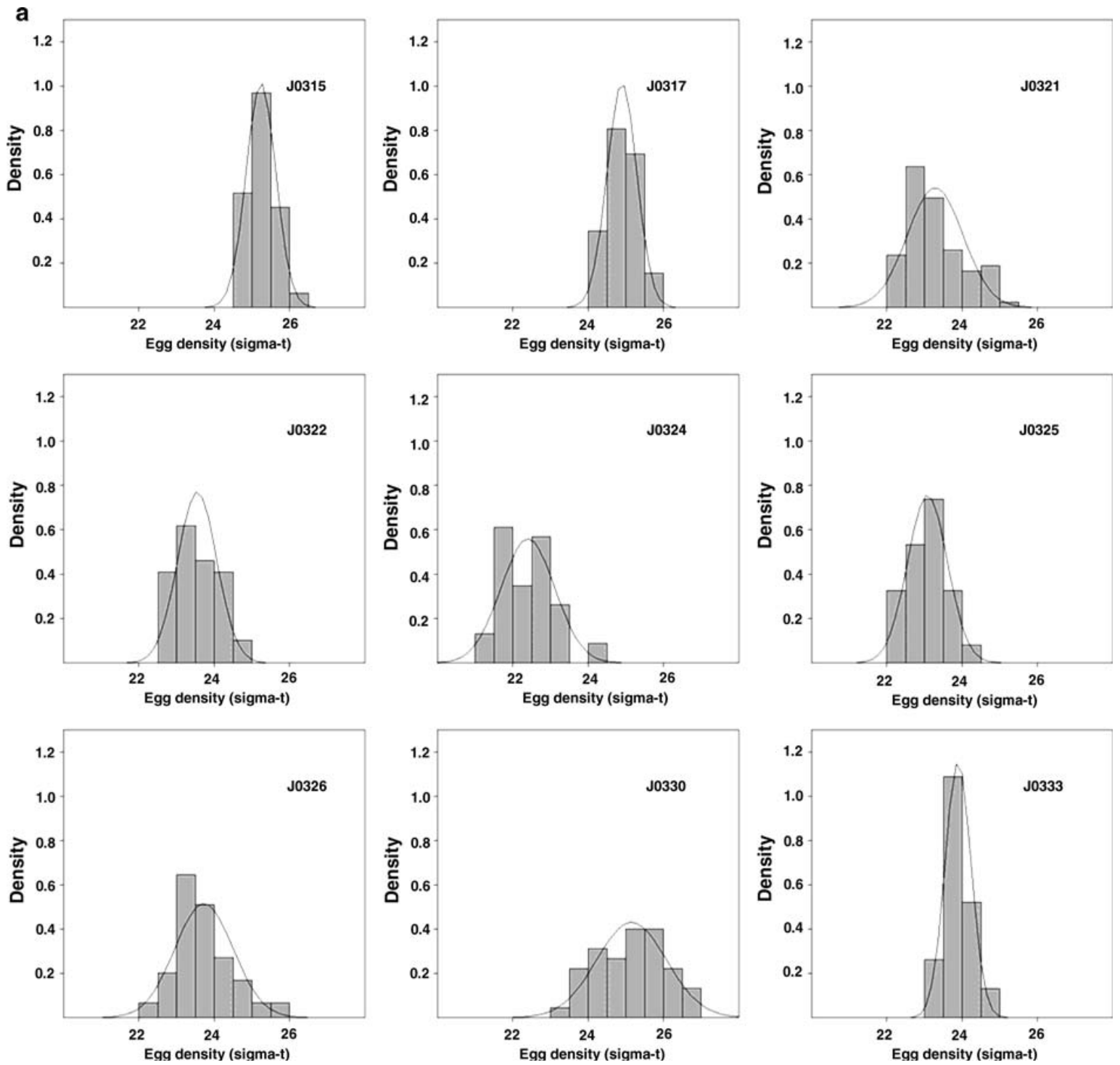

Figure 2a: Cumulative frequency distribution of anchovy egg density at each station with fitted Gaussian distribution in 2005. Stations referenced as in Table 1.

Figure 2b: Cumulative frequency distribution of anchovy egg density at each station with fitted Gaussian distribution in 2006. Stations referenced as in Table 1. 

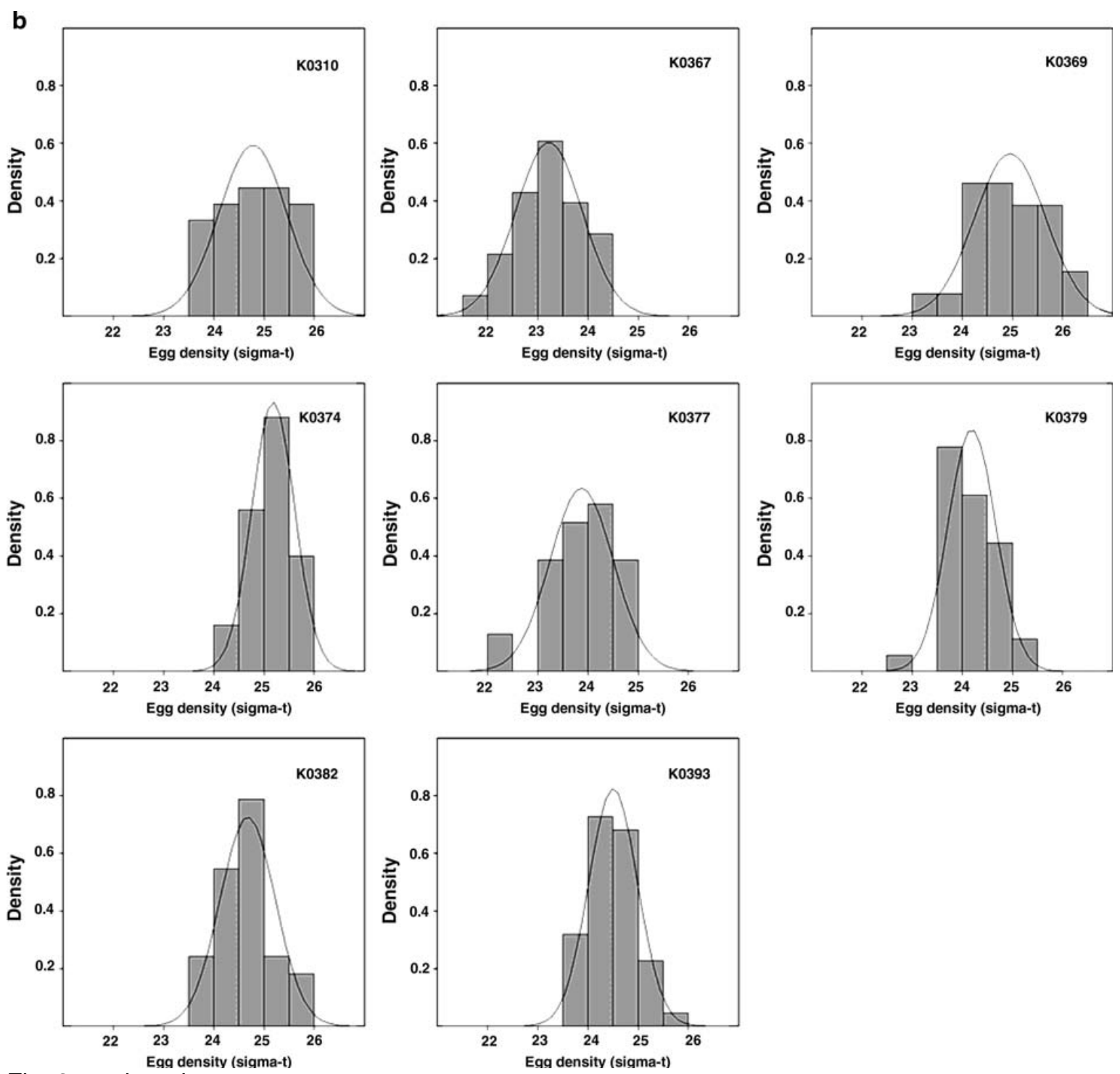

Fig. 2 continued 


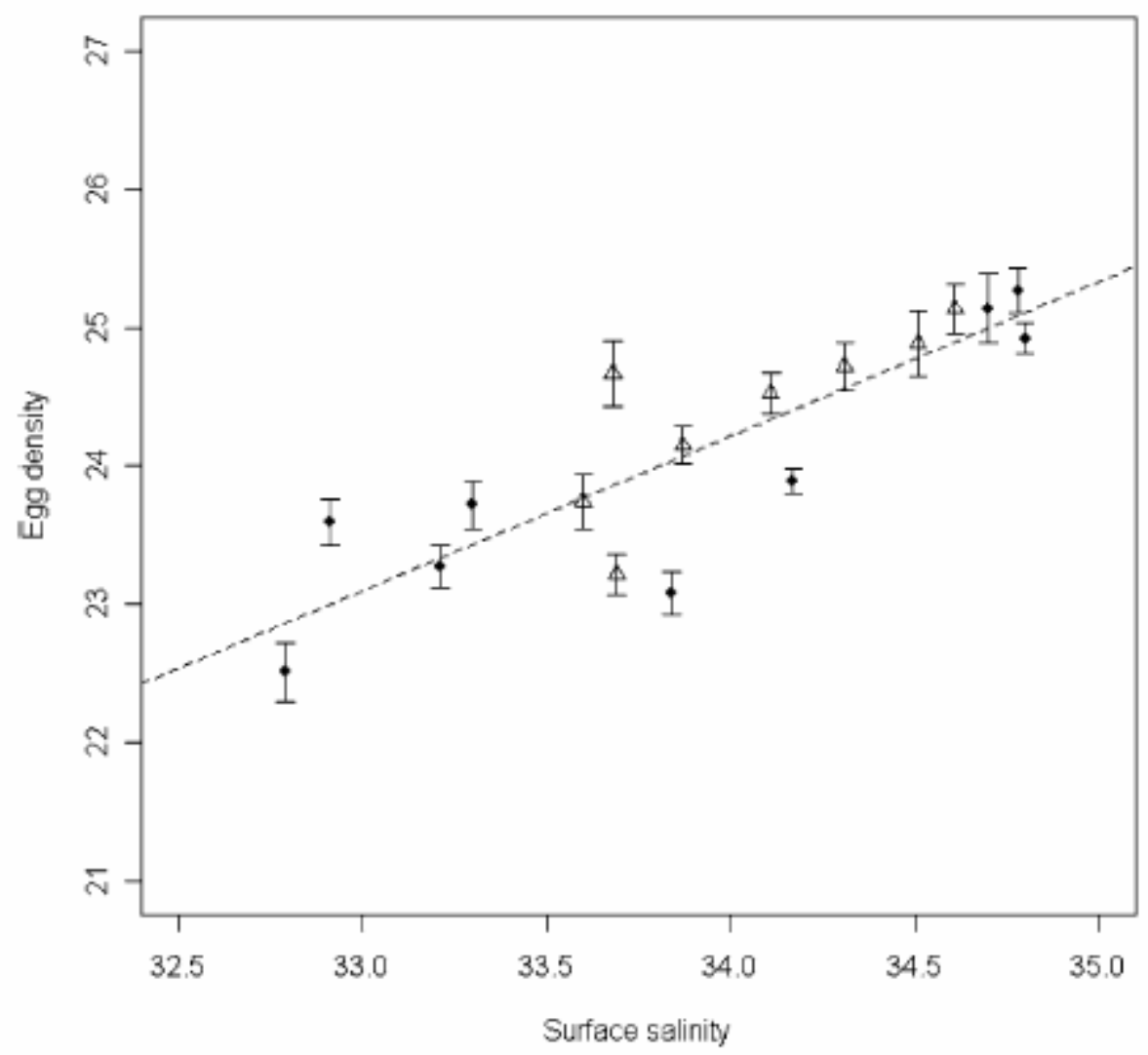

Figure 3: Regression of the at station mean anchovy egg density (sigma-t) on sea surface (3m) salinity. Points are the 2005 measurements and triangles the 2006 measurements. Bars indicate the $95 \%$ confidence interval of the Gaussian distribution in the individual egg distribution. The fitted dotted line is the linear regression (see Table 3): $E\left[\rho_{\text {egg }}\right]=-13.7919+1.1179 \times$ salinity . 


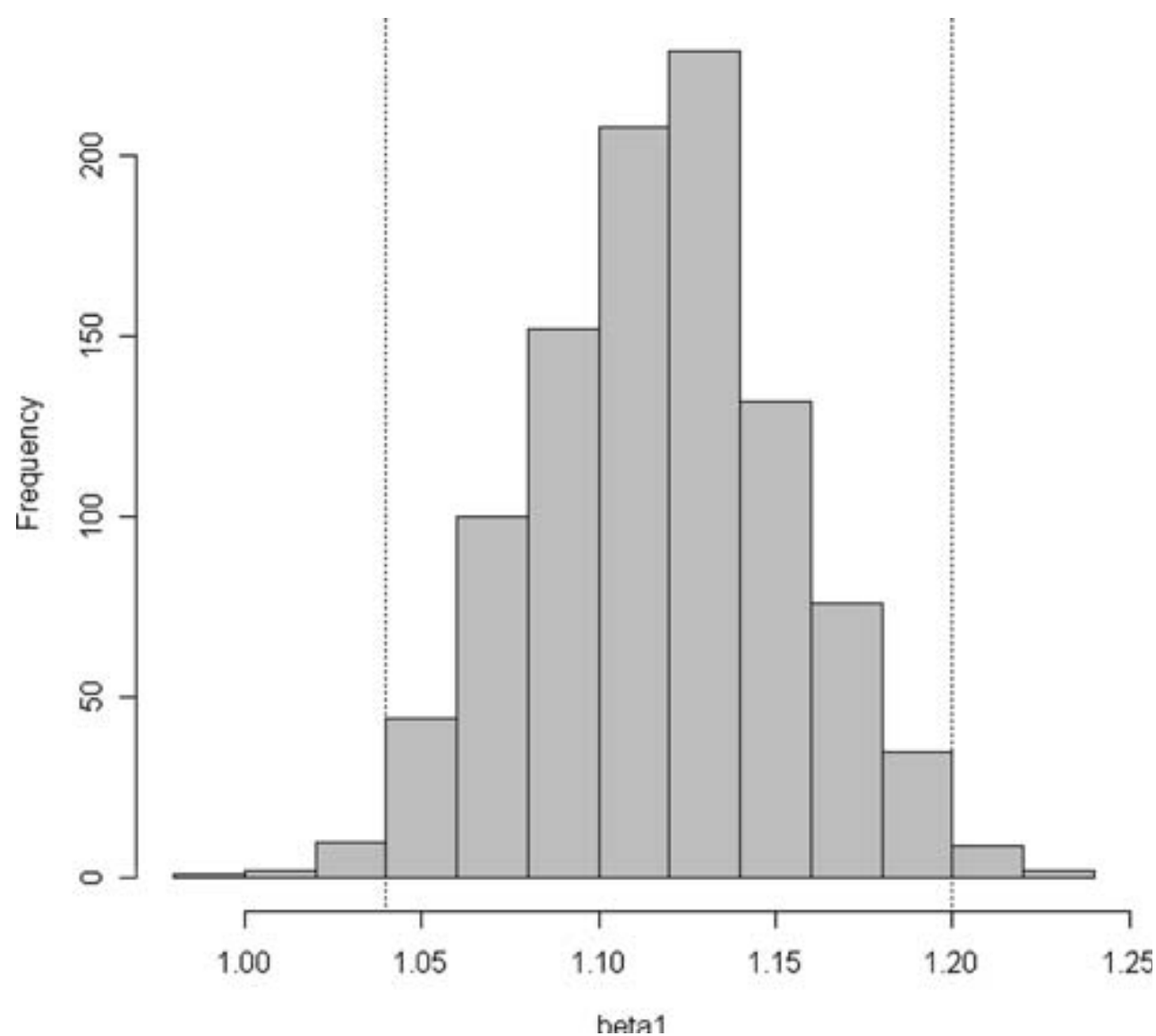

Figure 4: Bootstrap estimated frequency distribution of the slope parameter $\beta_{1}$ in the regression of mean egg density on sea surface salinity $\left(\bar{\beta}_{1}=1.119\right.$ and $\left.\sigma_{\beta 1}=0.04\right)$. Dotted vertical lines represent the $95 \%$ confidence limits. 